\title{
Nach der Abstimmung ist vor der Abstimmung
}

\section{Remo Osterwalder}

Dr. med., Mitglied des Zentralvorstandes der FMH, Departementsverantwortlicher Freipraktizierende Ärztinnen und Ärzte

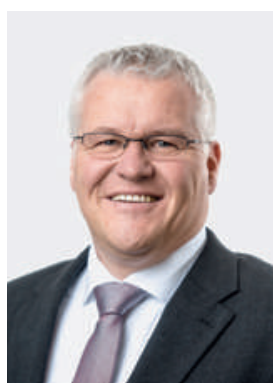

Wer sich durch das Abstimmungsresultat zur Volksinitiative «Für eine öffentliche Krankenkasse» im sicheren Hafen wähnt, interpretiert die Antwort der Stimmbürgerinnen und Stimmbürger falsch. Wurde die Initiative zur Einheitskasse im Jahre 2007 noch mit $71,2 \%$ abgelehnt, waren es am 28 . September 2014 "nur» noch 61,9\% bei gleicher Stimmbeteiligung. Ein solcher Rückgang von fast $10 \%$ ist relevant und könnte bei einer erneuten Vorlage mit überzeugenden Argumenten nochmals abnehmen oder gar zu einer Annahme einer Einheitskasse führen. Zwar haben die Versicherer somit noch einmal Zeit gewonnen, aber sicher nicht ihre Zukunft gesichert. Die zentralen Themen für die Versicherten wie etwa die freie Arztwahl oder ein gleichbleibender Leistungskatalog konnte die letzte Initiative nicht garantieren, weshalb die Chance für eine Annahme der Initiative eher klein war. Insbesondere die Aussage von Frau Nold, Direktorin von santésuisse, dass der Stimmbürger eine klare Unterstützung zum heutigen Versicherungssystem abgegeben habe, wirkt

\section{$38,1 \%$ der aktiven Stimmbürger sind gegen das heutige Versicherungssystem.}

beinahe etwas höhnisch: Klar freut sich jeder Prämienzahler auf die nächste Prämienerhöhung; klar macht es Sinn, Millionen von Reserven an der Börse anzulegen, um den nächsten Crash abzuwarten und dann einfach mit einer Prämienerhöhung beim BAG respektive bei den Bürgern anzuklopfen; klar freuen wir uns auf die netten, aber penetranten Werbeanrufe in der Vorweihnachtszeit. Es wäre an der Zeit, dass Frau Nold über die Bücher geht. Der Verdruss bei den Versicherten ist gross, aber das Misstrauen gegenüber einem Systemwechsel überwiegt momentan noch. Klare Zeichen kamen aus den Regionen, die gerne nach dem Staat rufen, wenn es mit den Verhandlungspartnern im Argen liegt. Gerade Kantone mit einem hohen Ja-Stimmenanteil haben interessanterweise einen hohen Kostenanstieg der spitalambulanten Leistungen. Gleichzeitig ist in Regionen mit eher hoher Ärztedichte ein Ja in die Urne gelegt worden.

\section{Referenzen}

1 Bundesamt für Statistik, Prämienentwicklung BL.

2 Daten santésuisse.
Bei staatlichen Eingriffen besteht jedoch ein Interessenkonflikt: Der Staat als Regulator, Anbieter von Gesundheitsdiensten und dann noch als Versicherer in der kantonalen öffentlichen Krankenkasse, das war wohl etwas zu viel für die Stimmbürgerinnen und Stimmbürger. Man weiss ja von breit angelegten Umfragen, dass das Vertrauen in die Politiker heutzutage nur noch sehr gering ist.

Das aktuelle Versicherungsmodell der Grundversicherung wird nicht überleben.

Es ist höchste Zeit, dass die Versicherer nun ein klares Zeichen setzen und längst anstehende Reformen angehen. Denn auch bei den Verwaltungskosten kam es zu einer Kostenexplosion. Hierzu ein Beispiel: In einem Kanton stieg die durchschnittliche Jahresprämie von 3276 CHF im Jahr 2005 auf 5160 CHF im Jahr 2013 [1]. Die sogenannten stabilen Verwaltungskosten von $5 \%$ nahmen im gleichen Zeitraum von $163.80 \mathrm{CHF}$ auf $258 \mathrm{CHF}$ zu. Dies entspricht einer Kostenexplosion von $58 \%$. Im gleichen Zeitraum nahmen die Kosten im ambulanten Sektor im entsprechenden Kanton aber "nur» um 38\% zu [2]. Bei den absoluten ProKopf-Kosten liegt die Erhöhung nur noch bei 30\%. Der Anteil der ambulanten Behandlungen an den Gesamtkosten ist in diesem Kanton von $28,4 \%$ im Jahr 2005 auf 24,9\% 2013 sogar gesunken. Dieses Beispiel illustriert eindrücklich, wie Zahlen einfach für die eigene Interpretationen zu gebrauchen sind, um damit vom eigentlichen Problem abzulenken.

Das Ausland ist beispielsweise immer überrascht, dass unser System die Finanzierung von Politikern mit Verwaltungsmandaten durch Prämiengelder zulässt. Solche Interessenkonflikte der Volksvertreter wären in anderen europäischen Demokratien undenkbar. Was wären denn die Voraussetzungen für einen Systemwechsel? Falls es gelingt, ein faires und transparentes Versicherungsmodell auf einer reellen Datenlage mit 100\% Abdeckungsgrad zu entwickeln, das einerseits das Konsumverhalten der Patienten sowie deren Risikoprofil widerspiegelt und andererseits die effektive Kostenentwicklung aufzeigen würde, ohne irgendwelche statistische Manipulationen - ich bin sicher, dann wäre der Stimmbürger für eine Änderung des Systems bereit.

Es braucht tiefgreifende Reformen und zwar rasch und nicht erst sechs Monate vor der nächsten $\mathrm{Ab}$ stimmung, die so sicher wiederkommen wird, wie das Amen in der Kirche. 